\title{
Evidence of independent introductions of RHDV2 strains in Poland based on the genome analysis of viral isolates from 2016-2018*
}

\author{
Andrzej Fitzner®, Andrzej Kesy, Krzysztof Bulenger and Wieslaw Niedbalski \\ Department of Foot and Mouth Disease, National Veterinary Research Institute, Zduńska Wola, National Veterinary Research Institute, Puawy \\ Poland
}

The aim of this study was the molecular epidemiology of independently introduced RHDV2 strains in Poland. The nucleotide sequences of RHDV2 diagnosed in domestic rabbits in 2018 in the voivodeships of Swietokrzyskie (strain PIN), Malopolskie (strain LIB) and Mazowieckie (strain WAK), and RHDVa from 2015 (strain F77-3) recognized in wild rabbits in Kujawsko-Pomorskie voivodeship were compared to the genome sequences of the first native RHDV2 strains from 2016-2017. The reference sequences available in public databases, the representative for a classical RHDV (G1-G5 genogroups), RHDVa (G6), non-pathogenic caliciviruses (RCV, GI.3 and GI.4) as well as original and recombinant RHDV2 isolates were included for this analysis. Nucleotide sequence similarity among the most distanced RHDV2 strains isolated in Poland in $\mathbf{2 0 1 8}$ was from $\mathbf{9 2 . 3 \%}$ to $\mathbf{9 8 . 2} \%$ in the genome sequence encoding ORF1, ORF2 and 3'UTR, between 94.8-98.7\% in the VP60 gene and between $91.3-98.1 \%$ in non-structural proteins (NSP) region. The diversity between three RHDV2 and RHDVa from 2015 was up to $16.3 \%$ in the VP60 region. Similarities are shown for the VP60 tree within the RHDV2 group, however, the nucleotide analysis of NSP region revealed the differences between older and new native RHDV2 strains. The Polish RHDV2 isolates from 2016-2017 clustered together with RHDV G1/RHDV2 recombinants, first identified in the Iberian Peninsula in 2012, while all strains from 2018 are close to the original RHDV2. The F77-3 strain clustered to well supported RHDVa (G6) genetic group, together with other Polish and European RHDVa isolates. Based on the results of phylogenetic characterization of RHDV2 strains detected in Poland between 2016-2018 and the chronology of their emergence it can be concluded that RHDV2 strains of 2018 and RHDV2 strains of 2016-2017 were introduced independently thus confirming their different origin and simultaneous pathway of spreading.

Keywords: RHDV2, RHDVa, phylogenetic analysis, VP60, non-structural genes, differentiation

Received: 19 November, 2020; revised: 07 January, 2021; accepted: 04 February, 2021; available on-line: 13 April, 2021

『e-mail: andrzej.fitzner@piwzp.pl

* Some results described in this paper were presented at 13th EPIZONE Annual Meeting „Breaking Walls”, 26-28 August 2019, Berlin, Germany

Acknowledgements of Financial Support: This study was supported by the Ministry of Science and Higher Education of Poland (Research project KNOW 2018/PIWet-PIB/LAB2/16).

GenBank Accession number of new nucleotide sequence reported in the manuscript: MN853658, MN853659, MN853660, MN853661

Abbreviations: RHDV, rabbit haemorrhagic disease virus (GI.1); RHDV2, rabbit haemorrhagic disease virus type 2 (GI.2); RHDVa, rabbit haemorrhagic disease virus, antigenic and genetic variant
(GI.1a); RCV, rabbit calicivirus (non-pathogenic); RCV-E1, European rabbit calicivirus 1 (non-pathogenic) (GI.3); RCV-A1, Australian rabbit calicivirus 1 and RCV-E2, European rabbit calicivirus 2 (nonpathogenic) (Gl.4)

\section{INTRODUCTION}

Rabbit Haemorrhagic Disease Virus (RHDV) is the cause agent of Rabbit Haemorrhagic Disease (RHD) - a fatal disease of wild and domestic European rabbits (Oryctolagus cuniculus), first detected in China in 1984 (Liu et al., 1984). At the end of the 1980s, RHD spread worldwide affecting many countries, mainly in Asia and Europe but also in Africa, Central and North America, Australia, and New Zealand (Abrantes et al., 2012). RHDV is a small, non-enveloped single stranded positive sense RNA virus of approximately $7.5 \mathrm{~kb}$ in length, belonging to genus Lagovirus, family Caliciviridae (Green et al., 2000). The RHDV genome is organized into two overlapping open reading frames (ORFs) (Meyers et al., 1991). ORF1 (nucleotides 10-7044) encodes a large polyprotein that is cleaved into several non-structural proteins (NSP) such as helicase, RNA-dependent RNA polymerase, protease, and a major structural capsid protein VP60. A minor structural protein, VP10, is encoded by ORF2 (nucleotides 7025-7378). The RHDV genome also encodes 5' and 3' untranslated regions (5'UTR and 3'UTR, respectively) (Meyers et al., 2000). In 19961997, the appearance of a new pathogenic RHDV form was diagnosed first in Italy, then in Germany (Capucci et al., 1998, Schirrmeier et al., 1999). This virus, an antigenic variant named RHDVa, dispersed rapidly across the world revealing its pandemic potential (Burmakina et al., 2016; Embury-Hyatt et al., 2012; Farnos et al., 2007; Grazioli et al., 2000; Le Gall-Reculé et al., 2003; Matiz et al., 2006; McIntosh et al., 2007; Oem et al., 2009; Tian et al., 2007). In 1996 non-pathogenic rabbit calicivirus (RCV) related to RHDV and providing total crossprotection to pathogenic RHDV has been detected in the duodenum of healthy rabbits (Capucci et al., 1996). Similar non-pathogenic rabbit caliciviruses providing no protection or partial cross-protection to RHDV infection were characterized in Australia (RCV-A1) and Europe (RCV-E1 and RCV-E2) (Strive et al., 2009; Le Pendu et al., 2017). Phylogenetic analysis of partial VP60 gene of more than 150 European RHDV strains (mainly French), collected between 1987 and 2003, revealed the presence of six genetic groups (genogroups G1 to G6) related to the year of isolation (Le Gall et al., 1998; Le Gall-Reculé et al., 2003, Novotny et al. 1997). Some of the classical RHDV strains that formed the G3 genogroup have gradually disappeared and evolved into two new groups 
- G4 and G5 (Le Gall-Reculé et al., 2003), while the RHDVa strains antigenically distinct from RHDV formed a homogeneous cluster - G6. Despite the antigenic and genetic differences, both RHDV and RHDVa induce infections with the same clinical manifestations and postmortem lesions. More recently, in 2010, a new RHDV form, originally designated as RHDV2 (also named RH$\mathrm{DVb})$, genetically and antigenically different from the classical RHDV and RHDVa variant, emerged in domestic and wild rabbits in France (Le Gall-Reculé et al., 2011) and subsequently in the other European countries, Australia, Africa, and Asia (Abrantes et al., 2013; Baily et al., 2014; Dalton et al., 2012; Duarte et al., 2015b; Hall et al., 2015; Hu et al., 2021; Isomursu et al., 2018; Le GallReculé et al., 2013; Neimanis et al., 2018b; Rahali et al., 2019; Westcott et al., 2014). Another pathogenic member of lagoviruses, morphologically similar to RHDV, is the European brown hare syndrome virus (EBHSV), responsible for the contagious and lethal disease of hares of the species Lepus europaeus and Lepus timidus, diagnosed first in Sweden in the early 80s (Gavier-Widén \& Mörner, 1991). Due to the gradual recognition of previously unknown pathogenic and non-pathogenic forms of rabbit and hare caliciviruses, a new classification of lagoviruses was proposed in 2017 (Le Pendu et al., 2017). According to this classification, RHDV2 genotype is designated as GI.2, the classic RHDV genotype as GI.1, and the individual genetic groups G1, G2, G3-G5 as GI.1b, GI.1c, GI.1d, respectively, while the subtype RHDVa (G6) is a GI.1a variant. In this classification system, the weakly pathogenic calicivirus MRCV and the non-pathogenic (NP) rabbit calicivirus RCV are unclassified while the other European and Australian NP rabbit caliciviruses form two distinct genotypes designated as GI.3 (European rabbit calicivirus 1, RCV-E1), and GI.4 (comprising Australian rabbit calicivirus 1, RCV-A1 and European rabbit calicivirus 2, RCV-E2). All these viruses are grouped within genogroup GI while genogroup GII contains the pathogenic EBHSV (genotype GII.1) and NP $\mathrm{HaCV}$ (genotype GII.2). For the sake of clarity and the consistency of the text referring to previous research on RHDV phylogenetics, the remainder of this paper will now use the old nomenclature to define the variants of lagoviruses.

In comparison to RHDV, RHDV2 is characterized with the distinctive pathogenic profile since it kills young rabbits (under 30 days of age) and rabbits previously vaccinated against RHDV as well as various hares' species, including the European hare (Lepus europaeus) and the mountain hare (Lepus timidus) (Dalton et al., 2012; Le Gall-Reculé et al., 2013; Le Gall-Reculé et al., 2017; Neimanis et al., 2018a; Puggioni et al., 2013; Velarde et al., 2017).

In Poland, the first cases of RHDV infections were confirmed in farming rabbits in 1988 (Gorski et al., 1988). Polish RHDV strains identified at that time were closely related to viruses isolated in Czech and Germany and are classified in the genetic group G2. In the following years, the disease spread rapidly among breeding rabbits, mainly on the small-scale farms. The new native RHDV isolates identified between 1994 and 2004 showed a genetic profile corresponding to European strains from G3-G5 groups (Fitzner \& Niedbalski, 2017). In the middle of the first decade of $21^{\text {st }}$ century, the RHDV strains exhibiting antigenic and genetic features like RHDVa variant were isolated in RHD outbreaks in Poland (Chrobocińska \& Mizak, 2007; Fitzner, 2009). RHDVa (G6) was the only representative of pathogenic RHDV continuously detected in Poland from 2004 to
2017 (Fitzner et al., 2012; Fitzner et al., 2017). The first Polish strains of RHDV2 were identified in 2016-2017 (Fitzner \& Niedbalski, 2018), and subsequent infections were confirmed in 2018. In the phylogenetic analysis of VP60 gene, the isolates RED 2016 and VMS 2017 clustered together with RHDV2 strains from France (1001), Spain (N11) and Portugal (CBVal16), while for the non-structural part of the genome both are grouped with the recombinant RHDV G1/RHDV2 viruses initially detected in the Iberian Peninsula (Lopes et al., 2015). These Polish RHDV2 isolates showed that about $97 \%$ of nucleotide sequence was identical with the European reference RHDV2 strains and approximately 18\% was different from classic RHDV and RHDVa variants (Fitzner \& Niedbalski, 2018).

The aim of this study was the molecular epidemiology of new RHDV2 strains from Poland which were collected during January - December 2018, unrelated to the previous cases. We also characterized the RHDVa strain confirmed in Poland for the first time in 2015 in wild rabbits, the population of which is residual, and the geographical distribution limited to separate enclaves (Krajewski \& Sadowski, 2013).

\section{MATERIALS AND METHODS}

\section{Specimens}

Tissue samples were collected in 2018 from cases with suspected RHD located in three different geographical regions of Central and Southern Poland, 150-350 km apart. The livers of two New Zealand White rabbits aged 4-5 months (sample PIN, Swietokrzyskie Voivodeship), and two mixed-breed rabbits 5-7 weeks old (sample LIB, Malopolskie Voivodeship) were taken from animals that died in small rural farms in January and November 2018. Furthermore, two dead pet rabbits have been sent from a veterinary clinic in December this year (samples WAK isolates 1 and 2, Mazowieckie Voivodeship, Warsaw). According to the attached information, the twoyear-old pet rabbit and the seven-year-old pet rabbit were from different owners and remained in the clinic periodically to continue treatment after previous tooth correction procedures. The treated animals died suddenly showing dyspnoea and restless movements immediately before death. Both pet rabbits have probably been infected through contact with the excreta of another sick animal previously housed in this clinic with no suspicion of RHD. At necropsy, the bloody discharge around the nostrils was observed in one rabbit. The livers of both rabbits were pale and friable demonstrating necrotizing features. The lungs demonstrated numerous petechial haemorrhages, and presence of uncoagulated blood was seen in the chest cavity. The liver homogenates of four wild rabbits (F77 1-4) found dead in the mid-northern region of Poland in Kujawsko-Pomorskie Voivodeship in 2015, that were collected for another study program and have not been previously diagnosed for RHD, were forwarded for this analysis.

\section{Virological diagnosis}

The hemagglutinating properties of the liver homogenates $(20 \% \mathrm{w} / \mathrm{v}$ in phosphate-buffered saline) of rabbits were assessed by HA test. The samples diluted 1:10 to $1: 20480$ in PBS ( $\mathrm{pH}$ 6.5) were incubated for $2 \mathrm{~h}$ at $2-8^{\circ} \mathrm{C}$ with $0.75 \%$ human group " 0 " red blood cells suspended in PBS. The same liver homogenates of rab- 
bits were screened using RHD-EBHS CR ELISA kit (code: 72575) for RHDV and then tested by differential ELISA kit (code: 80415$)$ to specify the type of RHDV antigen (RHDV, RHDVa, RHDV2). Both ELISA tests containing monoclonal antibodies were carried out in accordance with the manufacturer's instructions - OIE Reference Laboratory for RHD (IZSLER, Brescia, Italy). Briefly, in ELISA kit for antigen detection the wells of immunoplate ( 96 well, Maxisorb, Nunc) were coated (overnight at $2-8^{\circ} \mathrm{C}$ ) with RHDV-EBHSV positive/ negative $\mathrm{IgG}$ diluted in carbonate-bicarbonate buffer (0.05M, pH 9.6). After washing with PBS $0.05 \%$ Tween 20, $\mathrm{pH} 7.4$ (PBST), the samples of liver extracts diluted $1: 5$, and $1: 30$ in PBST with $1 \%$ of yeast extract (PBST$\mathrm{Y}$, dilution buffer) were incubated in duplicate for $1 \mathrm{~h}$ at $37^{\circ} \mathrm{C}$. After another washing step, the plates were incubated with cross-reactive (CR) pool of monoclonal antibodies (MAbs to detect uniformly all types of RHDVs and EBHSV) directly conjugated with horse-radish peroxidase (HRP), diluted in dilution buffer for $1 \mathrm{~h}$ at $37^{\circ} \mathrm{C}$. After the last washing step, o-Phenylenediamine (OPD) dissolved in citrate phosphate buffer $(0.15 \mathrm{M} \mathrm{pH}$ 5.0) activated with $\mathrm{H}_{2} \mathrm{O}_{2}$ was added to the wells and the reaction was stopped after 5 minutes with $1 \mathrm{M} \mathrm{H}_{2} \mathrm{SO}_{4}$ to read the absorbance of the samples (OD $492 \mathrm{~nm}$ ). To identify the type of RHDV antigen by using differential ELISA kit, the wells of immunoplate were coated with rabbit $\mathrm{IgG}$ anti- RHDV using standard carbonate buffer. After PBST washing step, the test and control samples were added to the coated wells with ELISA buffer (PBST-Y) Tween 20, pH 7.4. All the samples (except controls) were tested in two dilutions: 1:4, 1:25. After the $1 \mathrm{~h}$ incubation at $37^{\circ} \mathrm{C}$ with agitation, followed by washing with PBST the appropriate wells were incubated $(1 \mathrm{~h}$ at $37^{\circ} \mathrm{C}$ ) with specific (anti-RHDV, RHDVa, RHDV2) and cross-reactive monoclonal antibodies that react with each type of RHDV. In the next step, all the wells received anti-IgG mouse HRP conjugate. The presence of antigen was detected after a 5 min incubation with the substrate (OPD). The absorbance value (OD $492 \mathrm{~nm}$ ) of test samples were compared to the positive/negative controls and RHD reference viruses.

\section{RHDV, RHDVa, RHDV2 control antigens}

The reference viruses of native RHDV G2 (strain KGM 1988), RHDVa (strains: GRZ 2004, BBI 2017) from RHD outbreaks previously detected in Poland, recombinant VP60 protein in a form of virus-like particle (VLP) based on classical RHDV G2 (strain SGM 1988) genetic material (Gromadzka et al., 2006), two Polish RHDV2 strains (RED 2016, VMS 2017) and French RHDV2 13-69 strain of 2013 (received from ANSES, Ploufragan, France) were included for the analyses.

\section{Molecular analysis}

Total RNA was extracted from $100 \mu \mathrm{L}$ of $20 \%$ liver homogenate as described previously (Fitzner et al., 2012) and used for molecular tests. Two real-time RT-PCRs with primers and probes enabling discrimination between classic RHDV or RHDV2 were used for the detection of viral RNA and typing as described previously (Duarte et al., 2015a; Fitzner \& Niedbalski 2018; Gall et al., 2007). Conventional RT-PCR using the OneStep RT kit (Qiagen), and specific oligonucleotide primers have been applied to amplify the overlapping fragments of full RHDV genome as described previously (Fitzner \& Niedbalski 2018). The PCR products were gel-extracted and directly sequenced using ABI Prism BigDye ${ }^{\mathrm{TM}}$ ter- minator v3.1 Cycle sequencing kit and ABI3773x1 DNA sequencer (Applied Biosystems). The sequences of structural gene VP60 and non-structural protein (NSP) region of RHDV2 strains were determined after analysis of partial sequences available in form of a text file (.txt) and fluorograms file (.abi) and by the compilation of these partial nucleotide sequences. For comparative analysis and evaluation of the homology of RHDV strains, BLASTn software was used (Altschul et al., 1990). The nucleotide sequences of four Polish RHDV strains from 2018, RHDV F77-3 from 2015, native RHDV2 from 2016-2017, and reference sequences representing classic RHDV and RHDVa (genogroups G1-G6) from different geographical regions, non-pathogenic rabbit calicivirus (RCV, RCV-E1, and RCV-A1) as well as original RHDV2 and recombinant RHDV2 isolates were aligned by ClustalW algorithm in MEGA7 software (Kumar et al., 2016). The sequences of Polish strains of RHDV representative of each of the genetic groups were included for the analyses.

The phylogenetic trees of the VP60 gene sequences (nucleotide residues 5305-7044) and NSP region (nucleotide residues 10-5304) were constructed with MEGA 7 software (Kumar et al., 2016) using Neighbor-joining phylogenetic method. A total of 75 sequences for capsid gene and 61 sequences for NSP part of RHDV, RHDVa, RHDV2 and MRCV, RCV-A1 genomes were retrieved from GenBank (accession numbers and the country origin of the strains have been pointed out in Figs. 1 and 2). For the NSP fragment, the same data set as for VP60 region was not used due to the lack of some genomic sequences in the database. The European brown hare syndrome virus (EBHSV) (accession number Z69620) was used as an outgroup to root the tree. Pairwise distances between sequences were calculated with MEGA7 using the p-distance method.

\section{RESULTS}

HA analysis of the tested RHDV showed that 2018 RHDV2 strains PIN, LIB, WAK (isolate 1, isolate 2) exhibited hemagglutination activity with high titres in the range of 10240-20480. The isolates F77-1, F77-2, F773, F77-4 agglutinated human red blood cells type 0 with titres 160, 320, 10240, 320, respectively. CR ELISA results indicated that RHD virus antigen was detectable in liver samples of all dead rabbits from 2018 RHDV suspected cases (PIN, LIB, WAK 1-2), as well as in the specimens of four wild rabbits (F77 1-4) from 2015. In the differential ELISA, the samples tested of 2018, 2015, and control antigens of the relevant types of RHD viruses, reacted only with one type of MAbs, demonstrating a high absorbance with OD $492 \mathrm{~nm}>1.0$ (Fig. 3). The PIN, LIB and WAK samples were distinct from RHDV, RHDVa and reacted with MAbs RHDV2 and the mixture of MAbs (cross-reactive). The same reactivity was also observed for the RED 2016 and VMS 2017 strains, while F77-3 reacted with MAbs RHDVa and CR pool of MAbs.

Using real-time RT-PCR method, RHDV2 RNA was detected in all the tested samples from 2018 outbreaks (CT value 12 -15 for PIN, LIB, WAK isolates and no CT for F77 1-4 samples from 2015). The results of real-time RT-PCR assay using RHDV specific probe and primers confirmed the presence of RHDV RNA in four F77 samples (CT values 17, 19, 20, 21) and negative results for PIN, LIB and WAK samples. The results received by using conventional RT-PCR and direct se- 


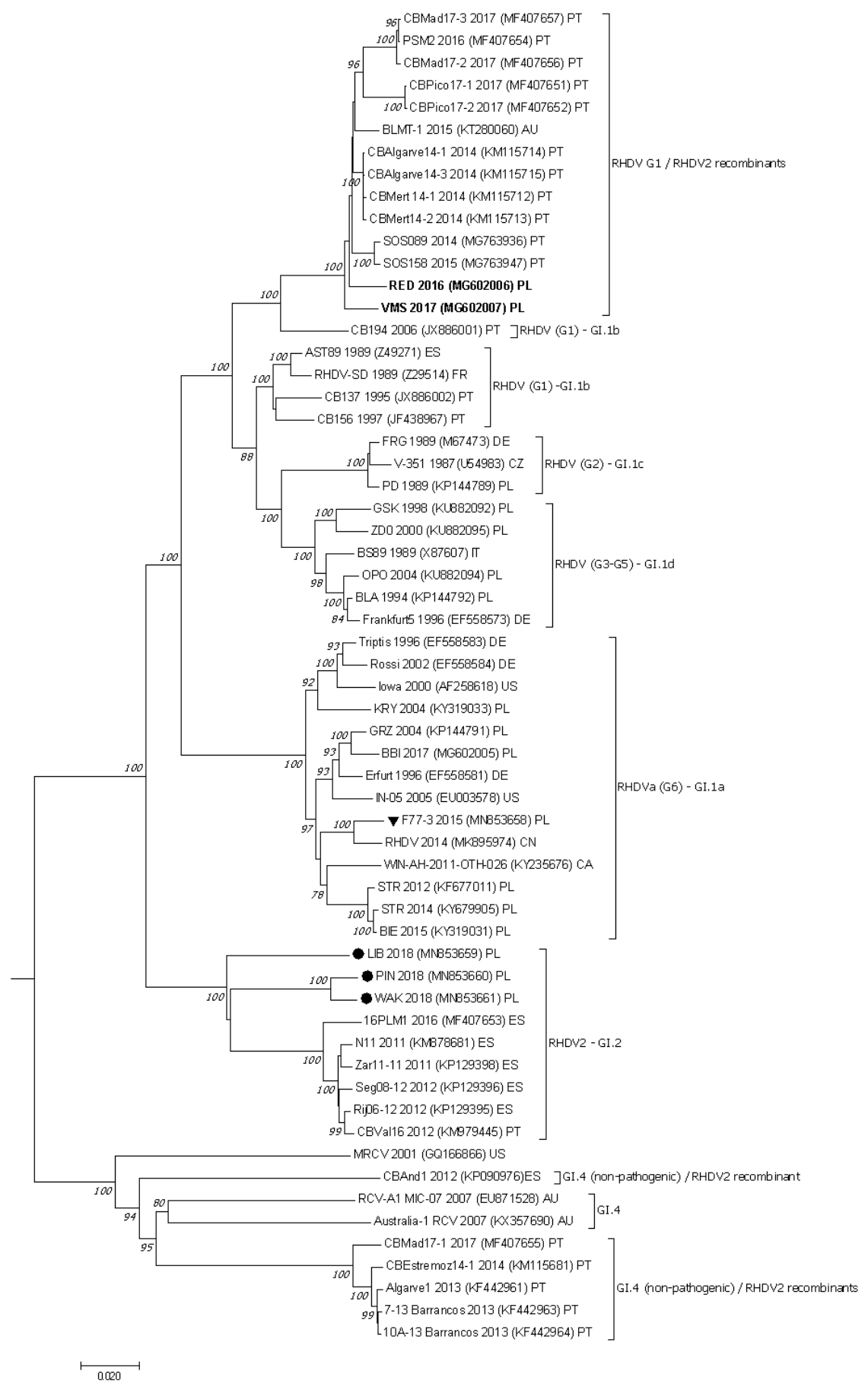

Figure1. Phylogenetic neighbor-joining tree for 75 capsid gene (VP60) sequences of pathogenic and non-pathogenic caliciviruses (nucleotides 5305-7044).

The European brown hare syndrome virus (EBHSV) was used as an outgroup to root the tree. RHDV major genetic groups defined by Le Gall and others (Le Gall et al., 2003) with the corresponding nomenclature according to Le Pendu and others (Le Pendu et al., 2017) are indicated next to the square brackets. RHDV2 $\bullet$ and RHDVa $\boldsymbol{\nabla}$ sequences established in this study (MN853658-61). Polish RHDV2 strains RED 2016 (MG602006), VMS 2017 (MG602007) are marked in bold. The percentage greater than 70\% of replicate trees in which the associated taxa clustered together in the bootstrap test (1000 replicates) are shown in italics above the major branches. The tree is drawn to scale, with branch lengths in the same units as those of the evolutionary distances computed in a p-distance method. Scale bare indicates the number of base differences per site. Evolutionary analyses were conducted in MEGA7 (Kumar et al., 2016). The accession numbers of the sequences retrieved from GenBank database are as follows: EF558573, JF438967, JX886001, JX886002, KC345612, KP144789, KP144792, KU882092, KU882094, KU882095, M67473, U54983, Y15427, X87607, Z29514, Z49271 for RHDV; AF258618, DQ069280, EF558581, EF558583, EF558584, EU003578, HQ917923, KF270630, KF677011, KJ814617, KP144791, KT003359, KY235676, KY319031, KY319033, KY679905, MG602005, MK895974 for RHDVa; FR819781, HE819400, KC345612, KC907712, KF442961, KF442963, KF962444, KM115712, KM115713, KM115714, KM115715, KM878681, KM979445, KM115681, KP090976, KP129395, KP129396, KP129398, KT280060, KT737803, LT168840, MF407651, MF407652, MF407653, MF407654, MF407655, MF407656, MF407657, MG763936, MG763947 for RHDV2; European and Australian non-pathogenic caliciviruses (RCV, RCV-E1, RCV-A1) are represented by X96868, AM268419, LT708122, EU871528 sequences, respectively, and the weakly pathogenic calicivirus MRCV by GQ166866 sequence. AU - Australia, CA - Canada, CN - China, CZ - Czech Republic, DE - Germany, ES - Spain, FR - France, IT - Italy, PT - Portugal, PL - Poland, RU - Russia, US - United States of North America. 


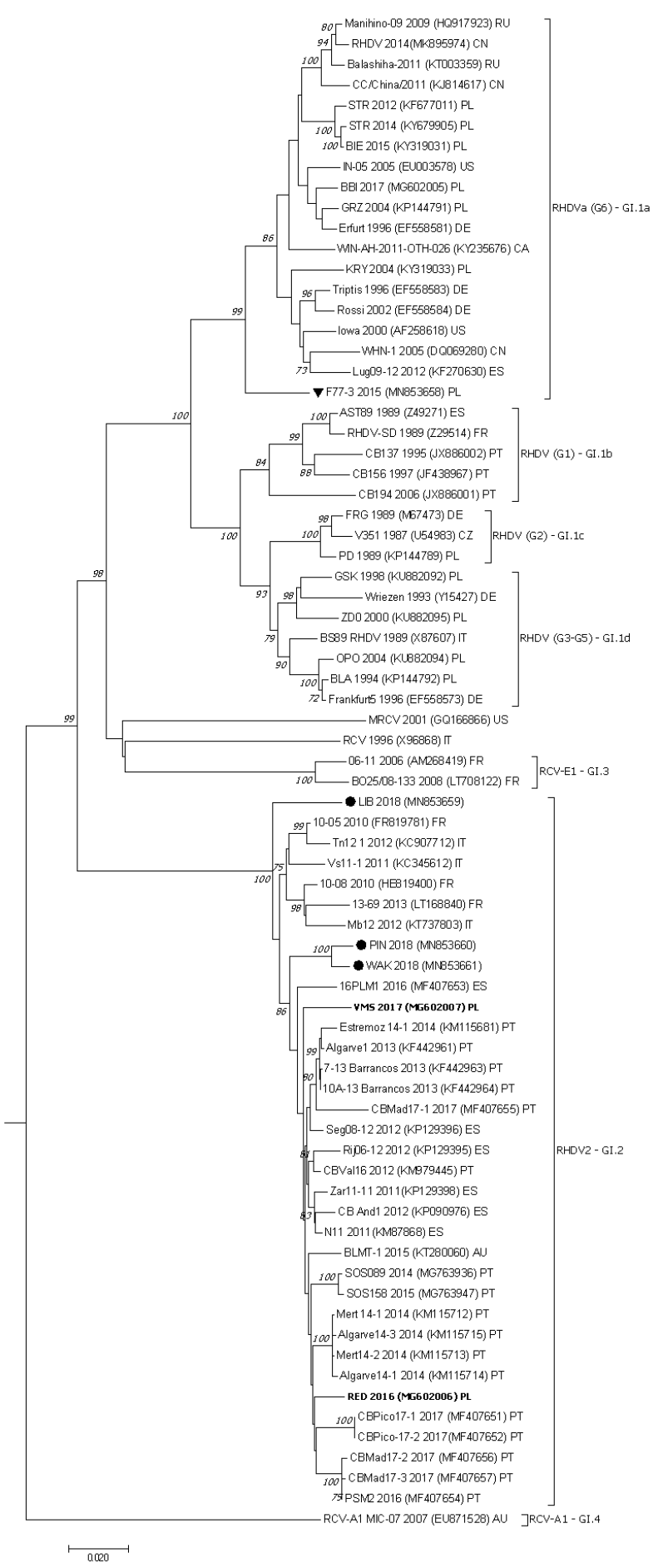

Figure 2. Phylogenetic neighbor-joining tree for the non-structural genes of 61 pathogenic and non-pathogenic caliciviruses (nucleotides 10-5304).

The European brown hare syndrome virus (EBHSV) was used as an outgroup to root the tree. RHDV major genetic groups defined by Le Gall and others (Le Gall et al., 2003) with the corresponding nomenclature according to Le Pendu and others (Le Pendu et al., 2017) are indicated next to the square brackets. RHDV2 - and RHDVa $\boldsymbol{\nabla}$ sequences established in this study (MN853658-61). Polish RHDV2 strains RED 2016 (MG602006), VMS 2017 (MG602007) are marked in bold. The percentage greater than 70\% of replicate trees in which the associated taxa clustered together in the bootstrap test (1000 replicates) are shown in italics above the major branches. The tree is drawn to scale, with branch lengths in the same units as those of the evolutionary distances computed in a p-distance method. Scale bare indicates the number of base differences per site. Evolutionary analyses were conducted in MEGA7 (Kumar et al., 2016). The accession numbers of the sequences retrieved from GenBank database are as follows: EF558573, JF438967, JX886001, JX886002, KP144789, KP144792, KU882092, KU882094, KU882095, M67473, U54983, X87607, Z29514, Z49271 for RHDV; AF258618, EF558581, EF558583, EF558584, EU003578, KF677011, KP144791, KY235676, KY319031, KY319033, KY679905, MG602005, MK895974 for RHDVa; KF442961, KF442963, KF962444, KM115712, KM115713, KM115714, KM115715, KM878681, KM979445, KM115681, KP090976, KP129395, KP129396, KP129398, KT280060, MF407651, MF407652, MF407653, MF407654, MF407655, MF407656, MF407657, MG763936, MG763947 for RHDV2; Australian non-pathogenic caliciviruses (RCV-A1) are represented by EU871528 and KX357690 sequences, and the weakly pathogenic calicivirus MRCV by GQ166866 sequence. AU - Australia, CA - Canada, CN - China, CZ - Czech Republic, DE - Germany, ES - Spain, FR - France, IT - Italy, PT - Portugal, PL - Poland, RU - Russia, US - United States of North America. 


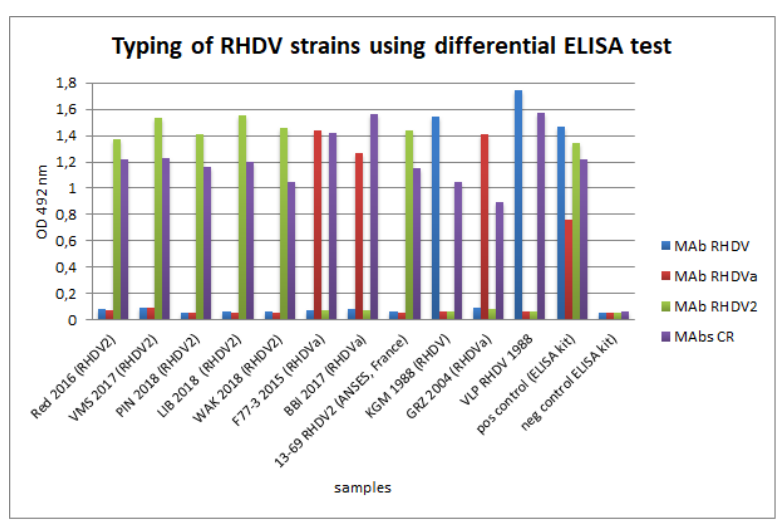

Figure 3. Typing of RHDV strains using differential ELISA test. Antigenic profiling of RHDV.

Liver homogenates from rabbits suspected of RHD (PIN 2018, LIB 2018, WAK 2018, F77-3 2015) and the controls samples were tested for reactivity with Mabs anti-RHDV2, anti-RHDVa, anti-RHDV and cross-reactive pool of Mabs (CR). Polish RHDV2 RED 2016, VMS 2017, RHDVa GRZ2004, RHDVa BBI 2017, French RHDV2 13-69 strains and RHDV VLP SGM 1988 were used as controls.

quencing of the amplified products confirmed the presence of RHDV2 in all liver samples taken from rabbits that have died in 2018, whereas genetic material of RHDVa variant was identified in F77 samples from 2015. Analysis of the nucleotide sequence of two RHDV isolates from two pet rabbits (WAK outbreak, December 2018) revealed $99.96 \%$ identity in the ORF1-ORF2 region (three synonymous changes). Out of these threepoint mutations two were placed in the NSP part of the genome and one in the VP60 region. For further analysis, the sequence of isolate 1 was used as a representative for WAK strain. In the case of RHDV strains obtained from wild rabbits of the Kujawsko-Pomorskie Voivodeship, genetic material extracted from the rabbit liver homogenate F77-3 with a highest HA titre was used. The partial genome sequences encoding ORF1, ORF2 and 3'UTR regions of three RHDV2 strains from 2018 (LIB, PIN, WAK) and RHDVa F77-3 strain from 2015 were determined by the sequencing of RTPCR amplification products and these sequences were submitted to GenBank (accession numbers: MN853659, MN853660, MN853661, MN853658, respectively). The RHDV2 genome sequences were of 7437 nucleotides (nt) in length for PIN and WAK from 2018, 7438 nt for LIB from 2018, and 7428 nt for RHDVa F77-3 strain. Nucleotide sequence similarity established in the BLAST program between the most distanced RHDV2 strains isolated in 2018 was $92.3 \%$ between PIN and LIB in the complete coding genome and 3'UTR (nt 10-7378), $95.1 \%$ and $91.3 \%$ between LIB and WAK in the VP60 gene and the NSP part of the genome (nt 10-5304). In contrast, the most territorially apart WAK and PIN strains showed the highest homology of $98.7 \%, 98.2 \%$ and $98.1 \%$ in the VP60 gene, the complete coding genome and 3'UTR and its NSP part, respectively. The sequence similarity between RHDV strains from 2018 and RHDV2 from 2016-2017 was 88\% in the complete coding genome and 3'UTR, $95-96 \%$ in VP60 and 85\% in NSP portion of the genome. Comparison of three VP60 nucleotide sequences of RHDV2 strains from 2018 with a sequence RHDVa F77-3 (2015) disclosed 16\% divergence, which was about $2 \%$ lower in relation to other Polish RHDVa isolates obtained between 2004-2017 from farmed rabbits (supplementary Table S1 at https:// ojs.ptbioch.edu.pl/index.php/abp/). In the NSP region, the differences between three 2018 strains and Polish RHDVa strains, including F77-3 isolate, were approximately 15\% (supplementary Table S2 at https://ojs. ptbioch.edu.pl/index.php/abp/). Phylogenetic analysis of VP60 gene confirmed that strains detected in 2018 belong to RHDV2 genotype (Fig. 1). The strains PIN, LIB and WAK clustered close to the reference RHDV2 strains of 2010-2013 detected in Italy and France, and near the Spanish RHDV2 PLM1 (MF407653) from 2016, reflected by 95-97\% nucleotide sequences identity. However, nucleotide analysis of the NSP region revealed differences between previous and new native RHDV2 strains (Fig. 2). RHDV strains from 2018 clustered to the original RHDV2 group but did not group with the Polish RHDV2 isolates from 2016-2017 which positioned together with RHDV G1/RHDV2 recombinants so far identified in the Iberian Peninsula and recently in Australia (Carvalho et al. 2017; Dalton et al., 2018; Hall et al., 2015; Lopes et al., 2015; Lopes et al., 2018). VP60 nucleotide sequence analysis showed the highest similarity of the F77-3 to the Russian RHDVa strains Manihino-09 (HQ917923) from 2009 (97.5\% identity), Balashiha-2011 (KT003359) (96.6\% identity), and to the Chinese RHDVa strain from 2014 (MK895974) (96.9\% identity). It is worth noting that the similarity between sequences F77-3 and Manihino-09 increased to $99.3 \%$ in the hypervariable $\mathrm{E}$ region of the capsid protein (Neill, 1992). In the NSP portion of the genome, $98.1 \%$ of nucleotide sequence identity was revealed for Chinese 2014 strain. As the genomic sequences of Russian RHDVa strains Manihino-09 and Balasiha-2011 are not available, there is no data concerning the similarity and their phylogenetic dependencies to F77-3 in NSP region. The similarity of the F77-3 isolate to six other RHDVa Polish strains coming from 2004-2017 ranged between 93.6\% for KRY 2004 (KY319033) to 95.6\% for GRZ 2004 (KP144791) in the capsid structural protein gene and between $94.6 \%$ for KRY 2004 to $96.1 \%$ for STR 2012 (KF677011) in the non-structural part of the genome. In the case of the BBI strain from 2017, these values in both regions were close to $95 \%$. Identity of F77-3 nucleotide sequence with German RHDVa strains Rossi 2002 (EF558584), Triptis 1996 (EF558583), American RHDVa Iowa 2000 (AF258618) strains was at $94.5 \%$ in the VP60 gene and about $95 \%$ in the NSP region. Phylogenetic analysis of the capsid protein gene and NSP region showed that F77-3 isolate from 2015 has clustered to well supported G6 genetic group (Fig 1. bootstrap value 99\% and Fig 2. bootstrap value $100 \%$ ), together with Polish, and other RHDVa strains from Europe, Asia, and North America.

\section{DISCUSSION}

Since the rabbit haemorrhagic disease virus (RHDV) was detected in China in 1984, three pathogenic forms of RHDV have been identified: classic RHDV (GI.1), the antigenic and genetic variant RHDVa (GI.1a), and the latest of them - RHDV2 (GI.2), also known as RH$\mathrm{DVb}$.

In this study, we presented three RHDV2 cases confirmed in both farmed and pet rabbits and RHDVa infection which occurred in native wild rabbits. The 2018 fatal infections in rabbits presented here do not have a spatial relationship, the samples come from three different regions of Poland and do not coincide when we analyze the time of onset. Two phylogenetically closest strains of RHDV2 from 2018, the PIN from the New 
Zealand rabbit outbreak in Swietokrzyskie Voivodeship, and WAK isolate detected in several-year-old pet rabbits (Mazowieckie Voivodeship) are nearly a year apart, with a geographical distance between the places of collection of about $150 \mathrm{~km}$. On the other hand, the most geographically distant strains, RHDV2 LIB isolated in mixed breed rabbits aged less than 2 months old in Malopolskie Voivodeship, and RHDV2 WAK, are separated by a distance of more than $350 \mathrm{~km}$ and a difference of 6 weeks between the dates of the first animal deaths. Likewise, the places of detection of RHDV2 strains from 2018 cannot be geographically linked to the origin of the first native RHDV2 strains, which were diagnosed in September 2016 in central Poland (Lodzkie Voivodeship), and in June of the following year near Szczecin in the far northwest region of the country (Fitzner \& Niedbalski, 2018). Both locations are more than $400 \mathrm{~km}$ away, and each of them is several hundred kilometres from the places where RHDV2 infections were diagnosed in 2018. Our investigations using ELISA with type-specific monoclonal antibodies showed that RHDV2 strains isolated over 2018 presented the same antigenic profile as native RHDV2 isolates of 2016-2017 and the reference RHDV2 French strain 13-69 (Fig. 3). On the other hand, based on the genetic characteristics of these strains and considering the chronology of their emergence, it can be concluded that the RHDV2 isolates from 2018 and RHDV2 from 2016-2017 were introduced independently thus confirming their different origin. The circulation of two viral forms which appeared in our country to unrelated cases is significant evidence confirming the parallel and independent spread of original RHDV2 and RHDV G1/RHDV2 recombinant over long distances, beyond the place of initial isolation. The new cases of RHD2 presented in this study occurred in rabbits of various breeds, different types of performance, in animals ranging in age from about one month to 7 years.

In turn, the case of RHDVa diagnosed in 2015 in wild rabbits completes the diverse picture of RHD infections. The occurrence of wild rabbit in Poland is associated with its introduction for hunting in the mid-nineteenth century. By some estimates, before World War II, the overall population of these animals in Poland reached 150,000 animals. In the following decades of the 20th century, their number decreased significantly due to severe winters and viral diseases such as myxomatosis and RHD. Currently, after many attempts at reintroduction, the total number of wild rabbits in our country is estimated at about fifteen thousand animals. Their occurrence is limited to small clusters, mainly in the central and western parts of the country (Krajewski \& Sadowski, 2013). RHD infections of wild rabbits living in Poland have not been previously confirmed. Thus, our results highlight a hitherto unknown active role of this small animal population in the transmission of RHD infections and its possible impact on virus survival outside farm rabbits.

Small but visible differences in the nucleotide sequence, amounting to $2-3 \%$ in a portion of the capsid gene noted between strain F77-3 and other Polish isolates of the RHDVa subtype, suggest its own evolutionary genetic path. This may be due to the spread of the virus in an airtight and highly isolated rabbit population that currently does not play a significant role in the native biocenosis, although all F77 specimens (1-4) have been submitted from the region inhabited by relatively large colonies of wild rabbits. For this reason, these animals can constitute, under our environmental conditions, a kind of specific reservoir in which the RHD virus can evolve differently than in farmed rabbits. The results of genetic analyses of the RHDVa F77-3 strain revealed the slightly higher variability of this isolate compared to the native RHDVa strains isolated over 13 years period in farm rabbits. The percentage of F77-3 VP60 nucleotide sequence variation relative to RHDVa GRZ2004 is twice as high $(4.4 \%)$ compared to relation between STR2014 and GRZ2004 (2.1\%) and three times higher as compared to BBI2017/GRZ2004 (1.1\%). The specific interaction between the host and the pathogen may likely have led to the survival of this somewhat different line of the RHDVa virus in the local ecosystem. Similar observations regarding the persistence of classical highly conserved RHDV strains of the G3-G5 genogroups, which were isolated for a period of 3 to 10 years, were made in our previous studies (Fitzner et al., 2012). It is worth noting that no other Polish isolate of RHDVa originating strictly from the Kujawsko-Pomorskie region has been genetically characterized in the past. The geographically closest sites where other RHDVa strains have been detected are situated approximately $200 \mathrm{~km}$ (strain BBI 2017), and $300 \mathrm{~km}$ (strain GRZ 2004) away. In this point of view, it should also be highlighted that a full comparison of the phylogenetic relationships of F773 strain with RHDVa viruses from Russia and China showing the closest similarity of the nucleotide sequences in the capsid gene was not possible due to the lack of available sequences covering the non-structural part of the genome.

Obtained results also indicate the ongoing process of competition or replacement of the previously dominant viruses - RHDVa or classic RHDV by RHDV2. This issue, like the conversion of RHDV to RHDVa, is delayed in Poland and probably throughout Central and Eastern Europe by several years compared to the situation observed in several other Western European countries with RHDVa (Burmakina et al., 2016; Matiz et al., 2006; Grazioli et al., 2000; Schirrmeier et al., 1999; Capucci et al., 1998). Identification of the RHDV F77-3 strain as a member of the RHDVa group also confirms our previous results indicating the exclusive presence of $\mathrm{RH}$ DVa in Poland since 2004 (Fitzner \& Niedbalski, 2017; Fitzner \& Niedbalski, 2018). In turn, the absence of new RHDVa cases diagnosed after 2017 may indicate the beginning of its gradual disappearance due to the emergence of RHDV2.

The results of our study reflect the dynamics of changes within the RHD virus isolated in Poland and, as it can be assumed, in other countries of Central and Eastern Europe. The presence of two types of RHDV2 strains confirms the infectious potential of these viruses and their ability to expand in new geographically distant areas. From an epidemiological point of view, the results presented in this paper have an important cognitive value and justify new research to follow the variability of the virus and its adaptability. In further research, it will be interesting to find an answer to the question of whether RHDVa will give way in the face of RHDV2 expansion and which form of RHDV2 - original, or perhaps some new type of recombinant - will gain the dominant position.

The results of phylogenetic studies and the variability of the studied RHDV strains indicate their diversity from previous native RHDV2 strains and the RHDVa subtype. The variety of viral forms which can appear at the same time and in the same area, as well as the revealed phenomena of recombination, support the continuation of epidemiological and genetic studies of RHD viruses, including complete genomic sequences. 


\section{Statement of author contributions}

$\mathrm{AF}, \mathrm{AK}, \mathrm{KB}, \mathrm{WN}$ have contributed to data collection, analysis, and writing the manuscript.

\section{Conflict of Interest}

The authors declare that they have no conflict of interest.

\section{Ethical approval}

This article does not contain any studies with animals performed by any of the authors.

\section{REFERENCES}

Abrantes J, van der Loo W, Le Pendu J, Esteves PJ (2012) Rabbit haemorrhagic disease (RHD) and rabbit haemorrhagic disease virus (RHDV): a review. Vet Res 43: 12. https://doi.org/10.1186/12979716-43-12

Abrantes J, Lopes AM, Dalton KP, Melo P, Correia JJ, Ramada M, Alves PC, Parra F, Esteves PJ (2013) New variant of rabbit hemorrhagic disease virus, Portugal, 2012-2013. Emerg Infect Dis 19: 19001902. http://dx.doi.org/10.3201/eid1111.050646

Altschul SF, Gish W, Miller W, Myers EW, Lipman DJ (1990) Basic local alignment search tool. J Mol Biol 215: 403-410. https://doi. org/10.1016/S0022-2836(05)80360-2

Baily JL, Dagleish MP, Graham M, Maley M, Rocchi MS (2014) RHDV variant 2 presence detected in Scotland. Vet Rec 174: 411. https://doi.org/10.1136/vr.g2781

Burmakina G, Malogolovkina N, Lunitsin A, Titov I, Tsybanov S, Malogolovkin A (2016) Comparative analysis of rabbit hemorrhagic disease virus strains originating from outbreaks in the Russian Federation. Arch Virol 161: 1973-1979. https://doi.org/10.1007/ s00705-016-2864-1

Capucci L, Fallacara F, Grazioli S, Lavazza A, Pacciarini ML, Brocchi E (1998) A further step in the evolution of rabbit haemorrhagic disease virus: the appearance of the first consistent antigenic variant. Virus Res 58: 115-126. https://doi.org/10.1016/ s011681702(98)00106-3

Capucci L, Fusi P, Lavazza A, Pacciarini ML, Rosi C (1996) Detection and preliminary characterization of a new rabbit calicivirus related to haemorrhagic disease virus but nonpathogenic. J Virol 70: 8614 8623. https://doi.org/10.1111/j.1439-0450.1996.tb00311.x

Carvalho CL, Silva S, Gouveia P, Costa M, Duarte EL, Henriques AM, Barros SS, Luis T, Ramos F, Fagulha T, Fevereiro M, Duarte MD (2017) Emergence of rabbit haemorrhagic disease virus 2 in the archipelago of Madeira, Portugal (2016-2017). Virus Genes 53: 922-926. https://doi.org/10.1007/s11262-017-1483-6

Chrobocińska M, Mizak B (2007) Phylogenetic analysis of partial capsid protein gene of rabbit haemorrhagic disease virus (RHDV) strains isolated between 1993 and 2005 in Poland. B Vet I Pulawy 51: 189-197

Dalton KP, Nicieza I, Balseiro A, Muguerza MA, Rosell JM, Casais R, Alvarez AL, Parra F (2012) Variant haemorrhagic disease virus in young rabbits, Spain. Emerg Infect Dis 18: 2009-2012. https://doi. org./10.3201/eid1812.120341

Dalton KP, Arnal JL, Benito AA, Chacon G, Martin Alonso JM, Parra F (2018) Conventional and real-time RT-PCR assays for the detection and differentiation of variant rabbit hemorrhagic disease virus (RHDVb) and its recombinants. J Virol Methods 251: 118-122. https://doi.org/10.1016/j.jviromet.2017.10.009

Duarte MD, Carvalho CL, Barros SC, Henriques AM, Ramos F, Fagulha T, Luis T, Duarte EL, Fevereiro M (2015a) A real time Taqman RT-PCR for the detection of rabbit hemorrhagic disease virus 2 (RHDV2). J Virol Methods 219: 90-95. https://doi.org/10.1016/j. jviromet.2015.03.017

Duarte M, Henriques M, Barros SC, Fagulha T, Ramos F, Luis T, Fevereiro M, Benevides S, Flor L, Barros SV, Bernardo S (2015b) Detection of RHDV variant 2 in the Azores. Vet Rec 176: 130. https://doi.org/10.1016/j.meegid.2015.08.005

Embury-Hyatt C, Postey R, Hisanaga T, Burton L, Hooper-McGrevy K, McIntyre L, Millar K, Pasick J (2012) The first reported case of rabbit hemorrhagic disease in Canada. Can Vet J 53: 998-1002. PMID: 23450867

Farnos O, Rodriguez D, Valdes O, Chiong M, Parra F, Toledo JR, Fernandez E, Lleonart R, Suarez M (2007) Molecular and antigenic characterization of rabbit hemorrhagic disease virus isolated in Cuba indicates a distinct antigenic subtype. Arch Virol 152: 1215-1221. https: / doi.org/10.1007/s00705-006-0926-5

Fitzner A (2009) Characterization and immunogenic properties of Polish strains of RHD virus. B Vet I Pulawy 53: 575-582
Fitzner A, Niedbalski W, Paprocka G, Kesy A (2012) Identification of Polish RHDVa subtype strains based on the analysis of a highly variable part of VP60 gene. Pol J Vet Sci 15: 21-29. https://doi. org/10.2478/v10181-011-0109-5

Fitzner A, Niedbalski W (2017) Phylogenetic analysis of rabbit haemorrhagic disease virus (RHDV) strains in Poland. Arch Virol 162: 3197-3203. https://doi.org/10.1007/s00705-017-3476-0

Fitzner A, Niedbalski W (2018) detection of rabbit haemorrhagic disease virus 2 (GI.2) in Poland. Pol J Vet Sci 21: 451-458. https://doi. org $/ 10.24425 / 122618$

Gall A, Hoffmann B, Teifke JP, Lange B, Schirrmeier H (2007) Persistence of viral RNA in rabbits which overcome an experimental RHDV infection detected by a highly sensitive multiplex real-time RT-PCR. Vet Microbiol 120: 17-32. https://doi.org/10.1016/j.vetmic.2006.10.006

Gavier-Widén D, Mörner T (1991) Epidemiology and diagnosis of the European brown hare syndrome in Scandinavian countries: a review. Rev Sci Tech 10: 453-458.

Gorski J, Mizak B, Mizak Z, Komorowski A (1988) Clinical and anatomopathological features of rabbits peste (viral haemorrhagic disease of rabbits). Życie Weterynaryjne 63: 266-269 (in Polish)

Grazioli S, Agnoletti F, Scicluna MT, Masoero N, Guercio A, Fallacara F, Lavazza A, Brocchi E, Capucci L (2000) Rabbit haemorrhagic disease virus (RHDV) subtype "A" (RHDVa) is replacing the original strain in some Italian regions. In Brocchi E, Lavazza A eds. pp. 202-203. Proceedings of the 5th International Congress of the European Society for Veterinary Virology, Brescia Italy.

Green KY, Ando T, Balayan MS, Berke T, Clarke IN, Estes MK, Matson DO, Nakata S, Neill JD, Studdert MJ, Thiel H-J (2000) Taxonomy of the Caliciviruses. J Infect Dis 181 (Suppl 2): S322-S330. https://doi.org/10.1086/315591

Gromadzka B, Szewczyk B, Konopa G, Fitzner A, Kesy A (2006) Recombinant VP60 in the form of virion-like particles as a potential vaccine against rabbit hemorrhagic disease virus. Acta Biochim Pol 53: 371-376. PMID: 16733562

Hall NR, Mahar JE, Haboury S, Stevens V, Holmes EC, StriveT (2015) Emerging hemorrhagic disease virus 2 (RHDVb), Australia. Emerg Infect Dis 21: 2276-2278. https://doi.org/10.3201/eid2112.151210

Hu B, Wei H, Fan Z, Song J, Chen M, Qiu R, Zhu W, Xu W, Xue J, Wang F (2021) Emergence of rabbit haemorrhagic disease virus 2 in China in 2020. Vet Med Sci 7: 236-239. https://doi.org/10.1002/ vms3.332

Isomursu M, Neimanis A, Karkamo V, Nylund M, Holopainen R, Nokireki T, Gadd T (2018) An outbreak of rabbit hemorrhagic disease in Finland. J Wildl Dis 54: 838-842. https://doi. org $/ 10.7589 / 2017-11-286$

Krajewski T, Sadowski J (2013) Attempts for introduction of rabbit Oryctolagus cuniculus by the method of small aviares. Stud Mat CEPL, Rogón 36: 174-183 (in Polish)

Kumar S, Stecher G, Tamura K. (2016) MEGA7: Molecular Evolutionary Genetics Analysis version 7.0 for bigger datasets. Mol Biol Evol 33: 1870-1874. https://doi.org/10.1093/molbev/msw054

Le Gall G, Arnauld C, Boilletot E, Morisse JP, Rasschaert D (1998) Molecular epidemiology of rabbit haemorrhagic disease virus outbreaks in France during 1988 to 1995. J Gen Virol 79: 11-19. https://doi.org/10.1099/0022-1317-79-1-11

Le Gall-Reculé G, Lavazza A, Marchandeau S, Bertagnoli S, Zwilgenstein F, Cavadini P, Martinelli N, Lombardi G, Guerin JL, Lemaitre E, Décors A, Boucher S, Le Normand B, Capucci L (2013) Emergence of a new lagovirus related to rabbit haemorrhagic disease virus. Vet Res 44: 81. https://doi.org/10.1186/1297-9716-44-81

Le Gall-Reculé G, Lemaitre E, Bertagnoli S, Hubert C, Top S, Decors A, Marchandeau S, Guitton JS (2017) Large-scale lagovirus disease outbreaks in European brown hares (Lepus europaeus) in France caused by RHDV2 strains spatially shared with rabbits (Oryctolagus cuniculus). Vet Res 48: 70. https://doi.org/10.1186/s13567-0170473-y

Le Gall-Reculé G, Zwilgenstein F, Boucher S, Le Normand B, Plassiart G, Portejoie Y, Decors A, Bertagnoli S, Guerin JL, Marchandeau S (2011) Detection of a new variant of haemorrhagic disease virus in France. Vet Rec 168: 137-138. https://doi.org/10. 1136vr.d697

Le Gall-Reculé G, Zwilgenstein F, Laurent S, de Boisseson C, Portejoie Y, Rasschaert D (2003) Phylogenetic analysis of rabbit haemorrhagic disease virus in France between 1993 and 2000, and the characterization of RHDV antigenic variants. Arch Virol 148: 65-81. https://doi.org/10.1007/s.00705-002-0908-1

Le Pendu J, Abrantes J, Bertagnoli S, Guitton JS, Le Gall-Reculé G, Lopes AM, Marchandeau S, Alda F, Almeida T, Célio AP, Bárcena J, Burmakina G, Blanco E, Calvete C, Cavadini P, Cooke B, Dalton K, Delibes Mateos M, Deptula W, Eden JS, Wang F, Ferreira CC, Ferreira P, Foronda P, Gonçalves D, Gavier-Widén D, Hall R, Hukowska-Szematowicz B, Kerr P, Kovaliski J, Lavazza A, Mahar J, Malogolovkin A, Marques RM, Marques S, Martin-Alonso A, Monterroso P, Moreno S, Mutze G, Neimanis A, NiedzwiedzkaRystwej P, Peacock D, Parra F, Rocchi M, Rouco C, Ruvoën-Clouet N, Silva E, Silvério D, Strive T, Thompson G, Tokarz-Deptula 
B, Esteves P (2017) Proposal for a unified classification system and nomenclature of lagoviruses. J Gen Virol 98: 1658-1666. https:// doi.org/10.1099/igv.0.000840

Liu SJ, Xue HP, Pu BQ, Quian NH (1984) A new viral disease in rabbits. Anim Husb Vet Med 16: 253-255

Lopes AM, Dalton KP, Magalhaes MJ, Parra F, Esteves PJ, Holmes EC, Abrantes J (2015) Full genomic analysis of new variant rabbit hemorrhagic disease virus (RHDVb) revealed multiple recombination events. J Gen Virol 96: 1309-1319. https://doi.org/10.1099/ vir. 0.000070

Lopes AM, Blanco-Aguilar J, Marton-Alonso A, Leitao M, Foronda P, Mendes M, Goncalves D, Abrantes J, Esteves PJ (2018) Full genome sequences are key to disclose RHDV2 emergence in the Macaronesian islands. Virus Genes 54: 1-4. https://doi.org/10.1007/ s11262-017-1523-2

Matiz K, Ursu K, Kesckemeti S, Bajmocy E, Kiss I (2006) Phylogenetic analysis of rabbit haemorrhagic disease virus (RHDV) strains isolated between 1988 and 2003 in eastern Hungary. Arch Virol 151: 1659-1666

McIntosh MT, Behan CS, Mohamed FM, Lu Z, Moran KE, Burrage TG, Neilan JG, Ward GB, Botti G, Capucci L, Metwally SA (2007) A pandemic strain of calicivirus threatens rabbit industries in the Americas. Virology J 4: 96. https://doi.org/10.1186/1743-422X-4-96.

Meyers G, Wirblich C, Thiel HJ (1991) Rabbit haemorrhagic disease virus-molecular cloning and nucleotide sequencing of a calicivirus genome. Virology 184: 664-676. https://doi.org/10.1016/00426822(91)90436-f

Meyers G, Wirblich C, Thiel HJ, Thumfart JO (2000) Rabbit haemorrhagic disease virus: genome organization and polyprotein processing of a calicivirus studied after transient expression of cDNA constructs. Virology 276: 349-363. https://doi.org/10.1006/ viro. 2000.0545

Neill JD (1992) Nucleotide sequence of the capsid protein gene of two serotypes of San Miguel sea lion virus: Identification of conserved and non-conserved amino acid sequence among calicivirus capsid proteins. Virus Res 24: 211-222. https://doi.org/10.1016/01681702(92)90008-W

Neimanis A, Ahola H, Larsson Petersson U, Lopes AM, Abrantes J, Zohari S, Esteves PJ, Gavier-Widen D (2018a) Overcoming species barriers: an outbreak of Lagovirus europaeus GI.2/RHDV2 in an isolated population of mountain hares (Lepus timidus). BMC Vet Res 14: 367. https://doi.org/10.1186/s12917-018-1694-7

Neimanis AS, Ahola H, Zohari S, Larsson Petersson U, Bröjer C, Capucci L, Gavier-Widén D (2018b) Arrival of rabbit haemorrhagic disease virus 2 to northern Europe: Emergence and outbreaks in wild and domestic rabbits (Oryctolagus cuniculus) in Sweden. Transbound Emerg Dis 65: 213-220. https//doi.org/10.1111/tbed.12650

Nowotny N, Bascunana CR, Ballagi-Pordany A, Gavier-Widen D, Uhlen M, Belak S (1997) Phylogenetic analysis of rabbit haemorrhagic disease and European brown hare syndrome viruses by comparison of sequences from the capsid protein gene. Arch Virol 142: 657673. https://doi.org/10.1007/s007050050109

Oem JK, Lee KN, Roh S, Lee KK, Kim SH, Kim HR, Park CK, Joo YS (2009) Identification and characterization of Rabbit Hemorrhagic Disease Genetic Variants Isolated in Korea. J Vet Med Sci 71: 1519-1523. https://doi.org/10.1292/jvms.001519

Puggioni G, Cavadini P, Maestrale C, Scivoli R, Botti G, Ligios C, Le Gall-Reculé G, Lavazza A, Capucci L (2013) The new French 2010 variant of the rabbit of the hemorrhagic disease virus causes an RHD-like disease in the Sardinian Cape hare (Lepus capensis mediterraneus). Vet Res 44: 96. https://doi.org/10.1186/1297-9716-44-96

Rahali N, Sghaier S, Kbaier H., Zanati A., Bahloul C (2019) Genetic characterization and phylogenetic analysis of rabbit hemorrhagic disease virus isolated in Tunisia from 2015 to 2018. Arch Virol 164: 2327-2332. https://doi.org/10.1007/s00705-019-04311-z

Schirrmeier H, Reimann I, Köllner B, Granzow H (1999) Pathogenic, antigenic and molecular properties of rabbit haemorrhagic disease virus (RHDV) isolated from vaccinated rabbits: detection and characterization of antigenic variants. Arch Virol 144: 719-735. https:// doi.org/ 10.1007/s007050050538

Strive T, Wright JD, Robinson AJ (2009) Identification and partial characterization of a new lagovirus in Australian wild rabbits. Virology 384: 97-105. https://doi.org/10.1016/j.virol.2008.11.004

Tian L, Liao J, Li JW, Zhou WR, Zhang XL, Wang HL (2007) Isolation and identification of a non-haemagglutinating strain of rabbit hemorrhagic disease virus from China and sequence analysis for the VP60 Gene. Virus Genes 35: 745-752. https://doi.org/10.1007/ s11262-007-0155-3

Westcott D, Frossard JP, Everest D, Dastjerdi A, Duff JP, Steinbach F, Choudhury B (2014) Incursion of RHDV2-like variant in Great Britain. Vet Rec 174: 333. https://doi.org/10.1136/vr.g2345

Velarde R, Cavadini P, Neimanis A, Cabezón O, Chiari M, Gaffuri A, Lavin S, Grilli G, Gavier-Vidén D, Lavazza A, Capucci L (2017) Spillover Events of Infection of Brown Hares (Lepus europaeus) with Rabbit Haemorrhagic Disease Type 2 Virus (RHDV2) Caused Sporadic Cases of an European Brown Hare Syndrome-Like Disease in Italy and Spain. Transbound Emerg Dis 64: 1750-1761. https://doi. org/10.1111/tbed.12562 